\title{
TEM Specimen Preparation of Partially-Embedded Electrodes From Proton Exchange Membrane Fuel Cell Membrane Electrode Assemblies
}

\author{
K.L. More and K.S. Reeves \\ Oak Ridge National Laboratory, Oak Ridge, TN
}

Proton exchange membrane (or polymer electrolyte membrane) fuel cells (PEMFCs) are being developed for future use as efficient, zero-emission power sources. The performance of PEMFCs, however, degrades during electrochemical aging, primarily as a result of microstructural, compositional, and/or chemical changes associated with the polymer membrane and/or the materials comprising the electrodes. Each of the electrodes are composed of several constituents which form interpenetrating, percolating (conducting) networks; a Pt (or Pt alloy) electrocatalyst supported on high surface area carbon material $(\mathrm{Pt} / \mathrm{C})$ provides electronic conductivity, a proton conducting ionomer surrounds the $\mathrm{Pt} / \mathrm{C}$ and holds the carbon support together, and 30-60vol.\% porosity provides for gas transport through the electrode. Room temperature (RT) ultramicrotomy (using a Leica UCT) has been used successfully to prepare epoxy-embedded TEM cross-sections from thin $(<100 \mathrm{~m})$, fragile, 3-layer membrane electrode assemblies (MEAs).[1,2] When the electrode structure is completely embedded with epoxy, imaging the Pt catalyst particles and carbon support within the electrodes is straightforward. Unfortunately, the presence of epoxy makes it virtually impossible to identify and characterize the continuous ionomer network that surrounds the $\mathrm{Pt} / \mathrm{C}$ network, which has long been an electrode constituent of primary interest. An embedding technique was required to "partially" embed the electrode such that the MEA would (1) hold together during RT ultramicrotomy and (2) allow for TEM imaging of unaltered pore surfaces and the ionomer network within the porous electrode.

The best way to start to prepare a partially-embedded electrode structure was to identify a commercial epoxy that was more viscous than those traditionally used for embedding samples for microtomy, which proved to be difficult. Most embedding techniques require an epoxy with a low viscosity to flow and completely fill the pores. Since the electrode structure is open (30-60 vol.\% porosity), all the epoxies evaluated resulted in fully embedded electrode structures. Partial curing of commercial embedding epoxies was a logical alternative, but was also not successful. Many of the epoxy materials required heat to cure, and when they were removed from the furnace or hot plate after different curing times, the viscosity of the epoxy changed and was difficult to control. The only epoxy that worked consistently for partially-embedding the MEA electrodes, and had an easily-controlled viscosity, was Gatan's G-1 epoxy (not normally used for microtome block preparation but used for traditional TEM sample preparation). To accomplish the partial-embedding of the electrodes, the G-1 was cured until it was slightly red in color (and the consistency of peanut butter). A thin layer was then spread across the top and bottom surfaces of the MEA, encasing it in a hard epoxy shell. The G-1-encased MEA was then set in a mold with Araldite 6005 epoxy to prepare a standard block for microtomy (cured at $\sim 60^{\circ} \mathrm{C}$ for $8 \mathrm{~h}$ ). SEM cross-section images of a partially-embedded electrode are shown in Fig. 1a and 1b. The surface of the electrode is embedded to a depth of $\sim 1-5 \mathrm{~m}$, enough for the G-1 to "hold on" to the electrode structure and keep it intact during microtomy. The electrode structure below this embedded surface layer is not embedded with any epoxy. A low magnification TEM image of an intact electrode from a microtomed section is shown in Fig. 2. The large-scale porosity remains completely intact and can be imaged (Fig. 3) revealing the nature of the pore surfaces and ionomer network (shown by arrows) within the electrode and the ionomer thickness and uniformity around Pt/C (Fig. 4). This technique is being applied to a variety of MEA systems as part of ongoing research to characterize the microstructure of aged MEAs.[3]

[1] D.A. Blom and J.R. Dunlap, Microsc. Microanal. 9(Suppl 2) (2003).

[2] K.S. Reeves, K.L. More, L.R. Walker, and J. Xie, Microsc. Microanal. 10(Suppl 2) (2004).

[3] Research sponsored by the U.S. Dept. of Energy, Office of Hydrogen, Fuel Cells, and Infrastructure Technologies Program, under contract DE-AC05-00OR22725 with UT-Battelle, LLC. 

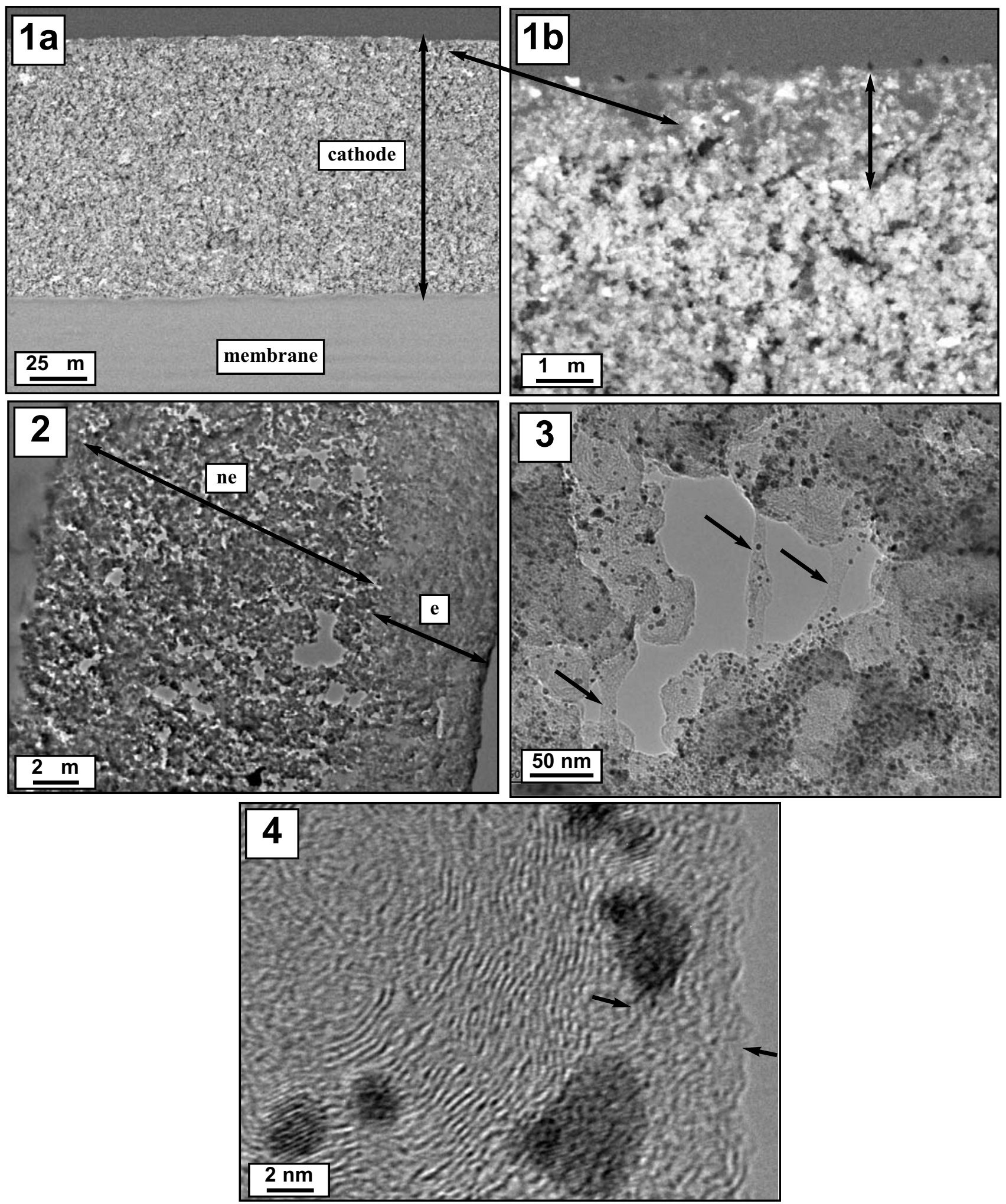

FIG. 1. SEM images of (a) partially embedded cathode and (b) embedded region near surface of cathode. FIG. 2. TEM image of intact cathode showing embedded (e) surface vs. non-embedded (ne) cathode. FIG. 3. TEM image of a pore in non-embedded electrode region showing $\mathrm{Pt} / \mathrm{C}$ and ionomer network. FIG. 4. TEM image of Pt (dark) catalyst particles on $\mathrm{C}$ support surrounded by ionomer (between arrows). 